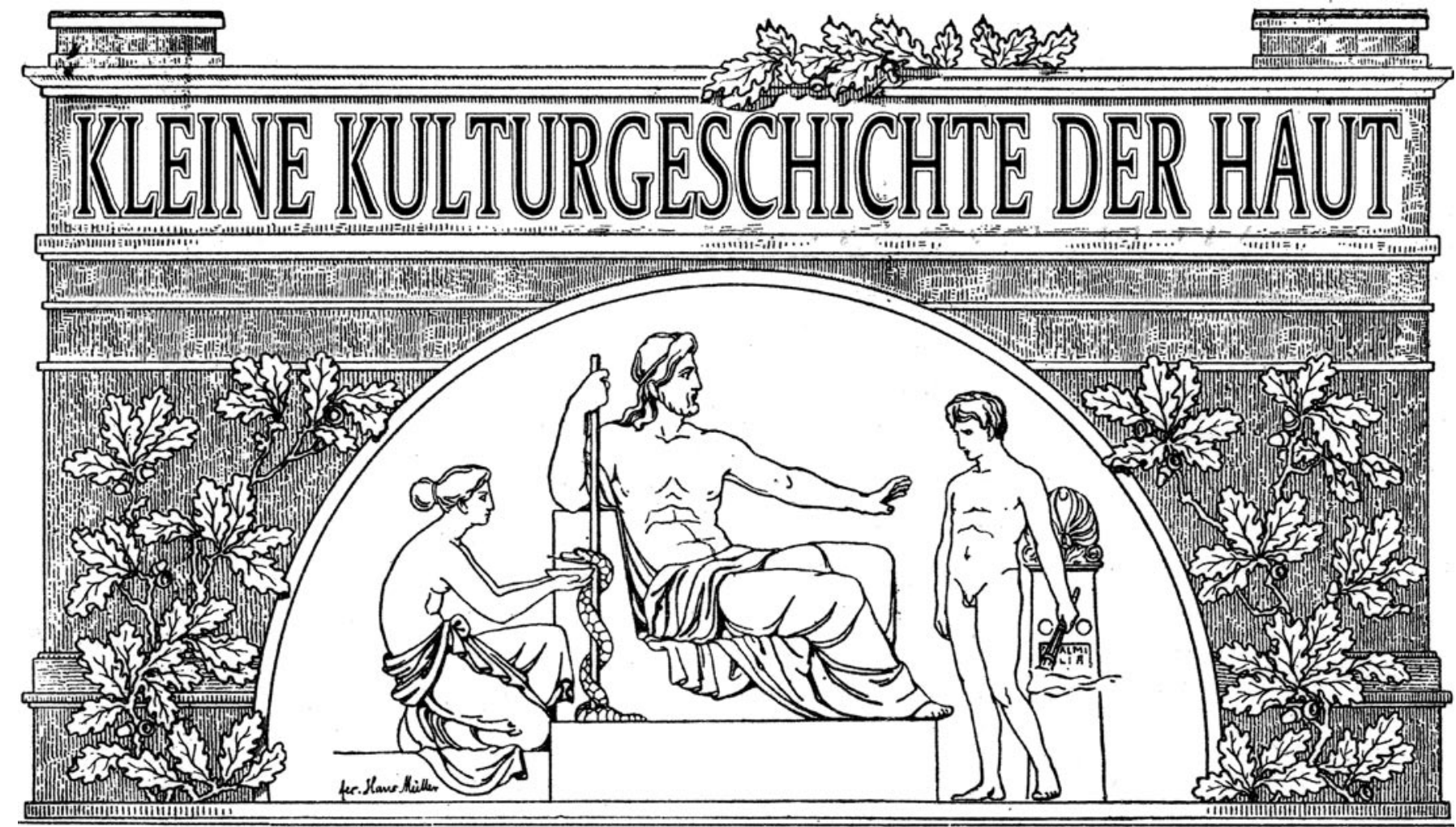

Die Zeitschrift „Aktuelle Dermatologie“ öffnet diese Rubrik und wird in loser Folge kurze, prägnante und informative Artikel darbringen zu vielen Aspekten der Kulturgeschichte in Beziehung zur Haut. Dies erstreckt sich auf alle Kulturen und Zeiten, auf die Künste, auf Mythen und Psychologie sowie natürlich auch auf medizinische Aspekte. Wir hoffen, dem geneigten Leser dadurch Interesse zu wecken, den Blick $z u$ weiten und Freude zu bereiten. Wir wünschen dieser Rubrik eine gute Aufnahme bei unseren Lesern und sind dankbar für Ihre Kommentare und Vorschläge für eine kleine Kulturgeschichte der Haut.

\title{
Xipe Totec (... der sich häutet, unser Herr) - ein Gott der Azteken
}

\section{K. Wegener}

Der Initiator der Rubrik „Kleine Kulturgeschichte der Haut“, der emeritierte Mannheimer Dermatologe E. G. Jung, hat die lose Folge der Artikel mit einer Arbeit „Vom Schinden“ eröffnet [1]. Darin macht er deutlich, dass in der abendländischen Mythologie und Historie Menschen ausschließlich zur Strafe und nie als Opfer der Götter geschunden worden seien.

Menschenhäutungen zu Ehren der Götter kannten aber die Kulturen Mesoamerikas vor Kolumbus' Entdeckung der Neuen Welt.

Die Spanier, die unter Führung des Hernán Cortés am 8. November 1519 als erste Europäer in Tenochtitlan, der Hauptstadt der Azteken, einzogen, waren konfrontiert mit ihnen zunächst unvorstellbaren Zahlen von Menschenopfern, die dieses Volk seinen Göttern während des Jahres bei den verschiedensten Festen darbrachte. Nur derartige rituelle Tötungen waren nach dem Glauben der Azteken geeignet, die gesamte irdische Ordnung aufrecht zu erhalten, denn ihre Mythen erzählten, das Selbstopfer der Götter habe die Welt entstehen lassen. Mit dem Opfern von Menschen dankten die Azteken den höchsten Wesen für ihr Selbstopfer und gaben gleichzeitig ihrem Glauben Ausdruck, nur durch Tod könne neues Leben entstehen.

Am eindrücklichsten manifestierte sich diese tiefe Überzeugung in dem Gott Xipe Totec (= der sich häutet, unser Herr). Er wurde meist als Mensch dargestellt und trug die abgezogene Haut eines Opfers über seinem Körper (Abb.1). Seine Verehrung war in verschiedenen postklassischen Kulturen Mesoamerikas weit verbreitet, und die Azteken hatten ihn von anderen Stämmen im Hochtal von Mexiko in ihr Pantheon übernommen. Wir sind über das Gottesbild des Xipe Totec bei den Azteken und den Ablauf des jährlich zu seinen Ehren gefeierten Festes Tlacaxipehuatlikli (Menschenhäutung) aus den Codices des Bernardino de Sahagún [2] und des Diego Durán [3] gut informiert ${ }^{1}$. Sahagún schildert das Aussehen des Gottes so:

\footnotetext{
${ }^{1}$ Die Schriften Sahagúns und Durans sind mehrfach übersetzt und interpretiert worden. Ich folge in dieser Arbeit den Transkriptionen und Interpretationen von B. Riese u. Mitarb. [4,7].
} 


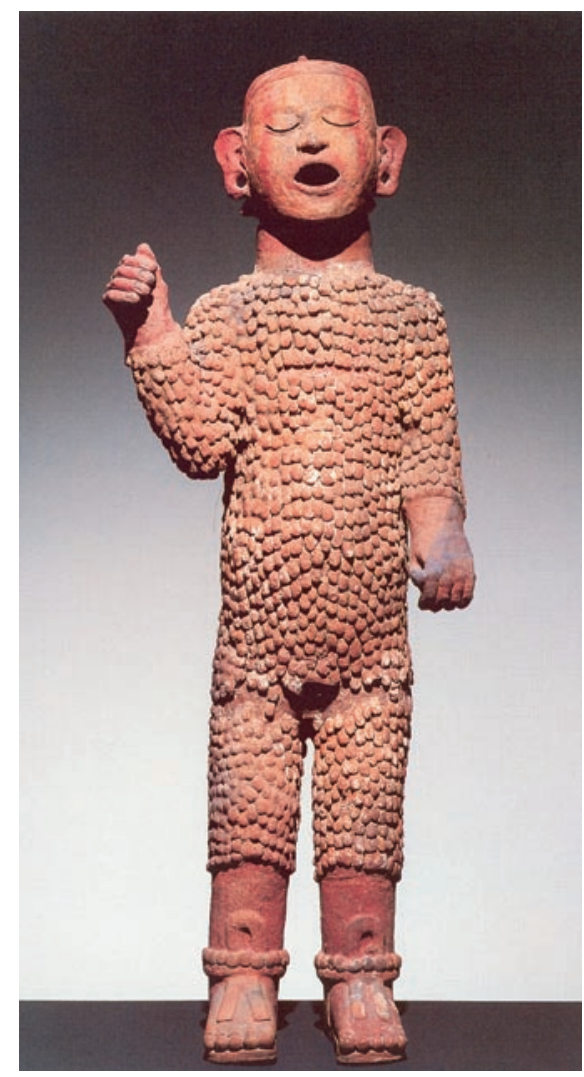

Abb. 1 Tonfigur des Gottes Xipe Totec.

Der Gott ist mit der abgezogenen Haut eines Opfers bekleidet, deren Struktur durch die "Schuppung“ deutlich wird. Besonders an Armen und Beinen erkennt man die „zweite Haut" aufgrund der Schnittränder ${ }^{2}$.

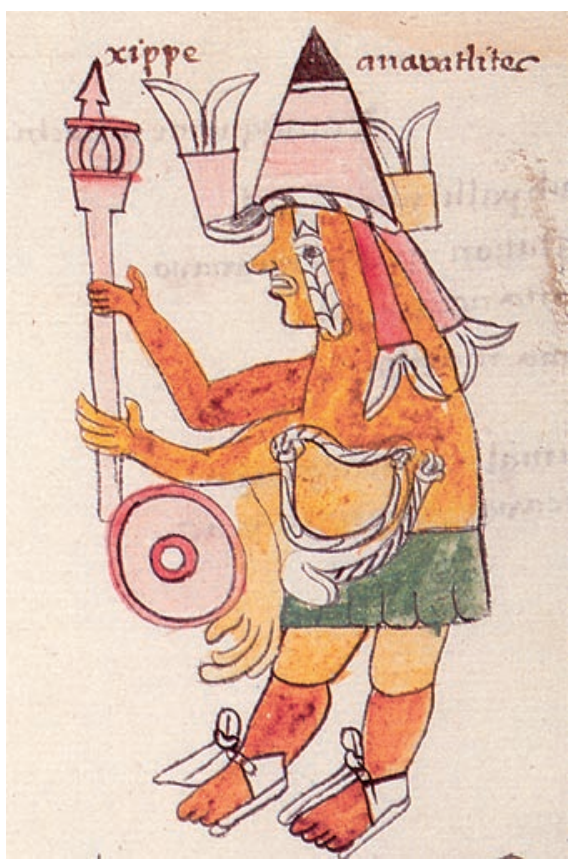

Abb. 2 Bild des Gottes Xipe Totec aus den „Primeros

Memoriales“ des Bernardino de Sahagún [5]. Zwischen dem Schild mit roten Kreisen und dem grünen Rock des Gottes sieht man die herabhängende „Hauthand" des Opfers.

ist: Xippe ycuic, totec (yoallavana)=Gesang für unseren Herren den Geschundenen. Eine in allen Passagen eindeutige Übersetzung und Interpretation ist sehr schwierig wenn nicht zur Zeit gar unmöglich. Deutlich aber geht aus den bisher klar zu entschlüsselnden Textteilen hervor, wie der Gott herbeigerufen und beschworen wird, das Land mit Regen zu tränken, wie nach dem Regen das Land ergrünt und der Mais reift. Dieses Lied weist Xipe Totec als Gott der Fruchtbarkeit aus.

Nach der Vorstellung der Azteken erstreckte sich die Welt in vier Grundrichtungen, die den vier Himmelsrichtungen entsprachen. Xipe Totec, auch Tlatlauhqui Tezcatlipoca genannt, der rote Tezcatlipoca, beherrschte den Osten, die Region des Sonnenaufganges, das männliche Viertel des Universums. Ihm wurden vielfältige Wirkungen und Fähigkeiten zugeschrieben:

er suchte Menschen heim mit verschiedenen Krankheiten

der Haut und der Augen,

Gottes in Sahagúns „Primeros Memoriales“ [5] (Abb. 2). Quilter [6] interpretiert diesen Hautmantel so: „Wie bei einem Goldüberzug oder dem Spelz eines Samenkorns liegt das wahre, lebendige Wesen des Gottes und seines menschlichen Ausdrucks unter der Oberfläche, bereit, in neues Leben auszuschlagen“. Der Rasselstab (Chicahuaztli), von dem hier geredet wird, ist ein Attribut des Gottes, aus dem er Samen verstreut. Xipe Totec verkörperte die Oberfläche der Erde und deren Kraft zu stetiger Erneuerung und gehörte mit Wasser, Samen und Sonne zum Kreislauf dieser Erneuerung.

Riese gibt in seinem Text „Aztekische Religion und Kunst“ [7] ein Lied wieder, das vermutlich am Fest des Gottes gesungen worden

\footnotetext{
2 Alle Abbildungen stammen aus dem Bildarchiv von B. Riese, Bonn.

${ }^{3}$ Ursprünglich war Xipe Totec eine Gottheit der Zapotec (Tzapotec)- und Yopi-Indianer, zweier der damals etwa 500 Völker auf dem Gebiet des heutigen Mexiko in den noch heute existierenden Gebieten Guerrero und Oaxaca.
}

er schuf im Frühling neues Leben der Pflanzenwelt,

er war der Gott der Goldschmiede,

und die Azteken hielten ihn für den Erfinder des Krieges.

An seinem Fest Tlacaxipehualitli (Menschenhäutung) wurde ihm eine große Zahl Gefangener geopfert. Ein Priester in Gestalt des Gottes - mit einer Menschenhaut wie ein Kleid über seinen Leib gezogen und somit Stellvertreter des Gottes - schnitt dem lebenden Opfer das Herz aus der Brust (Abb.3). Aus dem momentan vollgebluteten Thorax sog er das Blut in ein Rohr und hielt Herz und Blut der Sonne entgegen, um sie zu „baden“ und zu „speisen“. Ein Helfer trug danach auf die Lippen aller Götterbilder im Tempelbezirk Blut auf, damit diese davon kosteten. Diese Götterspeisung hatte den Sinn, die Fortsetzung des Lebens auf der Erde sicherzustellen (s. o.). Die Körper der toten Opfer wurden über die Treppenstufen des Tempels auf dessen Vorterrasse gestürzt. Dort nahmen die Ältesten der Stadtbezirke sie in Empfang, brachten sie in das Gemeindehaus, schunden sie und verteilten das Fleisch zum Ver- 


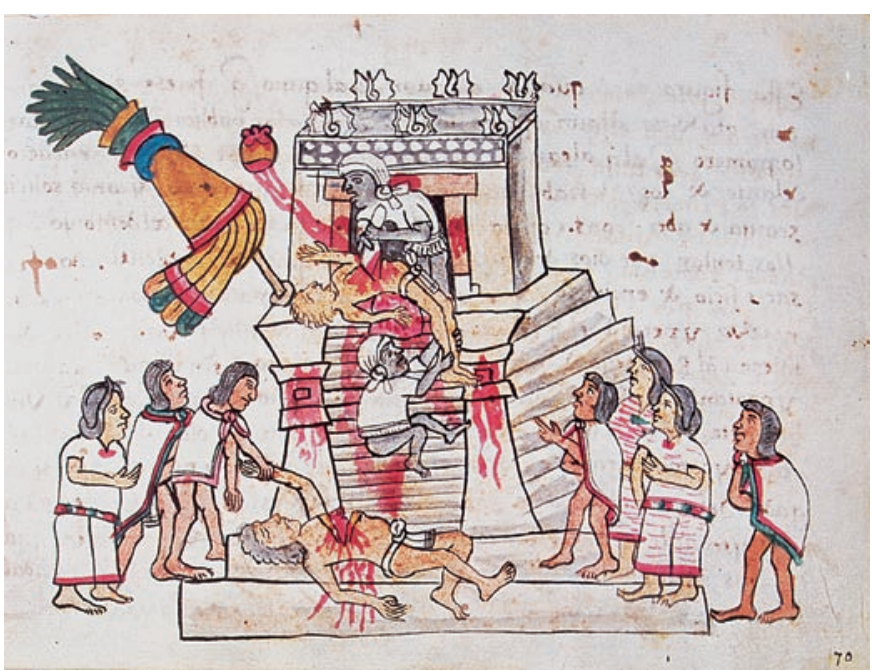

Abb. 3 „Herzopfer“ aus dem „Libro de la Vida“ [11]. Vor dem Eingang zum Tempel liegt ein Mensch, dem der Priester mit einem Steinmesser das Herz aus der Brust geschnitten hat. Es „schwebt“ in der Luft über dem Kopf des Opfers. Die Stufen zum Tempel sind blutbeschmiert, am Fuß des Tempels liegt ein zweites, totes Opfer.

zehr an die Mitglieder der Gemeinde ${ }^{4}$. Die abgezogenen Häute aber legten sich Männer um, die der Gott mit Hautulzera, Krätze, eiternden Augen, Wucherungen der Hornhaut und anderen Leiden geschlagen hatte. Sie eilten durch die Straßen der Stadt, triefend von Fett und besudelt mit Blut. Den Häuten der dem Gott Geopferten wurde gewissermaßen eine Emanationswirkung und Heilkraft zugesprochen. Betrunkene, Possenreißer und alle, die richtige Männer sein wollten, folgten ihnen. Frauen dagegen, die mit gleichen Leiden behaftet waren, brachten dem Gott ein Opfer an seinem Festtag. Legten Priester und Kranke die übergestreifte Haut ab, kam darunter der „neue Mensch“ zum Vorschein. Opfer und Tod hatten dann zu neuem Leben geführt. Diese Auffassung, dass es ohne Tod kein neues Leben geben könne, spiegelt sich deutlich auch im Bilde der sich häutenden Schlange, die den Azteken als Symbol der Wiedergeburt, der Fruchtbarkeit und des Überflusses galt. Ob aus diesen Vorstellungen die Assoziation des Gottes Xipe Totec mit der „Gefiederten Schlange“ (Quetzalcóatl) hervorgegangen ist, ist unklar [8].

Archäologen haben vor allem in Mexiko-Stadt Statuen des Gottes oder Verkörperungen von ihm (Priester, Krieger, Gefangene) gefunden, die - wie von Sahagún beschrieben - ein Kleid aus einer zweiten Haut tragen, das mit Schnüren über dem Kopf und auf dem Rücken zusammengezurrt ist. Hinter dem geöffneten Mund und den Augenschlitzen werden die entsprechenden Körperteile des Trägers sichtbar. Ein horizontaler Saum über dem Thorax macht die Stelle deutlich, an der das Herz entfernt und die danach vernäht worden war (Abb. 4).

Um 1900 hat man in der Umgebung des Templo Mayor in MexikoStadt einen Behälter für abgezogene Häute gefunden. Seine Außenfläche erinnert mit Aufrauungen an abgezogene Menschenhaut, die Priester und andere Verehrer des Gottes sich überhäng-

\footnotetext{
${ }^{4}$ Kannibalismus war in mehreren Kulturen des alten Mexiko üblich.
}

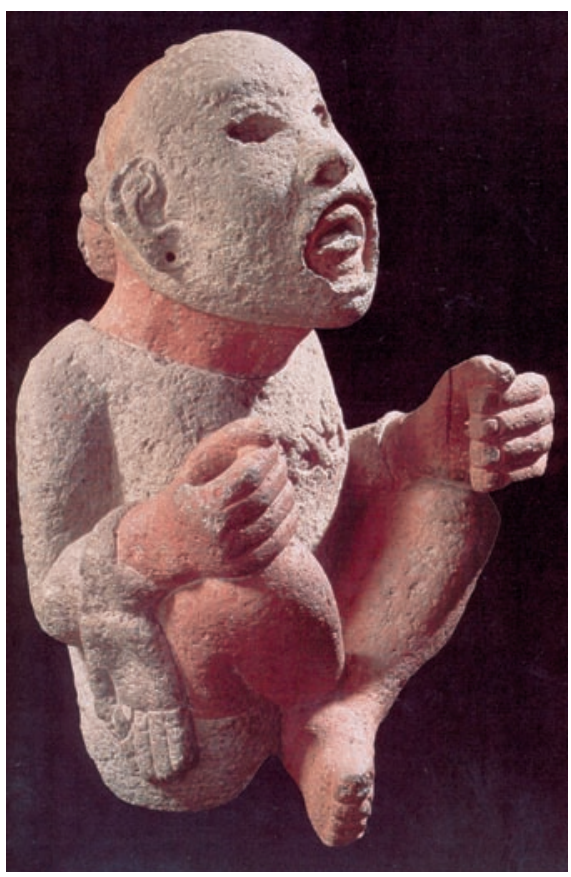

Abb. 4 Skulptur des Xipe Totec, bekleidet mit einer abgezogenen Haut. Im geöffneten Mund und am Hals sind die Körperteile des Trägers sichtbar.

ten, um sich der Gottheit anzuverwandeln. Nach Schilderungen von Sahagún haben sie die Häute im Festmonat 20 (!) Tage getragen und dann wahrscheinlich in solchen Behältern verwahrt.

Die Religion der Azteken kannte zwei weibliche Pendants zu Xipe Totec: die Fruchtbarkeitsgöttin Xilonen (wörtlich: Junges Maisrohr) - Göttin der ersten Maisfrucht der Saison - und Chicome Coatl (wörtlich: Sieben Schlange) - Göttin des Maissaatgutes. Beim Reinigungsfest Ochpa(na)nitzli (wörtlich: Fegen des Weges) ${ }^{5}$, das zu Ehren mehrerer Gottheiten veranstaltet wurde, war einer der Höhepunkte die Enthauptung und Häutung einer Frau durch einen Priester zu Ehren der Göttinnen Xilonen und Chicome Coatl. Der Priester trug Kleidung und Kopfschmuck einer der beiden Göttinnen, der er sich somit anverwandelte. Wie beim Fest für Xipe Totec legten sich die Priester Teile der Haut des Opfers $a^{6}{ }^{6}$. Der Gott und die Göttinnen der Fruchtbarkeit machen deutlich, wie wichtig Landwirtschaft, das Gedeihen der Saaten und die Reifung der Früchte für das tägliche Leben und Überleben in dieser Kultur waren. Diese Gottheiten entschieden in den Augen der Azteken über reiche Ernten oder Missernten.

Xipe Totec war auch der Gott der Goldschmiede, der angesehensten Handwerkerzunft in Mesoamerika. Es fehlt in der einschlägigen Literatur nicht an Interpretationen, Xipe Totecs Wirken als Lebensspender - und die damit verbundenen Rituale - mit seiner Verehrung durch die Goldschmiede in Verbindung zu bringen. So

\footnotetext{
${ }^{5}$ Riese macht in seinem Text zur Altmexikanischen Heilkunde und Gesundheitspflege [12] darauf aufmerksam, dass häufiges Fegen der gestampften Böden in Haus und Hof eine zentrale Arbeit im Dienste der häuslichen Hygiene darstellte, dass das Fegen im Tempeldienst eine religiöse Pflicht mit hohem Symbolgehalt darstellte und das Fest ochpananitzli (das Fegen des Weges) in diesem Zusammenhang gesehen werden müsse.

${ }^{6}$ Im Codex Borbonicus (Codex Borbonicus, Faksimile, Graz 1974) ist eine Opferszene dargestellt mit einem Priester im Zentrum, der die Gesichtshaut des Opfers als Maske trägt [8].
} 
macht Riese [7] auf eine mögliche Assoziation bei den Azteken zwischen der Farbe des Goldes und der der menschlichen Haut aufmerksam, die Xipe Totec übergestreift wurde. Diese Interpretation hat viel für sich, wenn man bedenkt, dass die von Priestern und anderen übergestreiften Häute geschundener Opfer gelb gefärbt waren und Teocuitlaquemitl (wörtlich: Goldenes Kleid) genannt wurden [9]. Auch die Formung goldener Hohlfiguren sowie das Überziehen von Schmuckstücken mit Goldblech hätten die Azteken möglicherweise analog zum Überziehen der Haut bei dem Gott gesehen.

Eine zweite interessante Interpretation gibt Moctezuma [10]. Er beschreibt ausführlich eine Halskette aus dem Templo Mayor in Mexiko-Stadt und geht auf die Technik des Metallgusses bei den Azteken zur Herstellung solcher Schmuckstücke ein. Die Azteken kannten das Gießen in verlorener Form. Bei diesem Verfahren werden mehrere Materialschichten der Form abgetragen oder ersetzt, und erst ganz zum Schluss wird das fertige Schmuckstück sichtbar. Diese Technik erinnere - so Moctezuma - an die Bearbeitung des Bodens in der Landwirtschaft, wo aus den Schichten der zunächst trockenen und dann bearbeiteten Erde grünes, fruchtbares Land werde. So nehme es nicht Wunder, dass der Gott der Fruchtbarkeit und des Ackerbaus - Xipe Totec - auch der Schutzgott der Goldschmiede sei.

Am 13. August 1521 übergab der letzte Aztekenkönig, Cuanktemoc, dem Spanier Cortés die Hauptstadt Tenochtitlan nach langer und auf beiden Seiten verlustreicher Belagerung. Die Spanier machten die Stadt dem Erdboden gleich. Götterfiguren, Kunstgegenstände, Pyramiden, Paläste wurden zerstört. Der Wiederaufbau war begleitet von der religiös-geistigen Eroberung des Reiches durch die Franziskaner, Augustiner und Dominikaner. Den überlebenden Azteken fiel es schwer, sich zu ihren alten Göttern zu bekennen, waren sie doch in den Kämpfen gegen die Spanier von ihnen völlig im Stich gelassen worden. Auch wurde religiöser Widerstand gegen das Christentum von den Eroberern brutal unterdrückt.

\section{Danksagung}

Herrn Prof. Dr. Bertold Riese, Direktor des Instituts für Altamerikanistik und Ethnologie der Universität Bonn, und seinen Mitarbeitern danke ich herzlich für Literatur, Bildmaterial, Hinweise und Diskussionen.

\section{Literatur}

1 Jung EG. Vom Schinden. Akt Dermatol 2004; 30: 81 - 84

2 de Sahagún B. Florentine Codex: A General History of the Things of New Spain. Books 1-12, Anderson AJO, Dibble CE (Hrsg). Santa Fe: 1950-1982

${ }^{3}$ Durán D. Historia de las Indias de Nueva Espana e islas de tierra firme. Garibay AM (Hrsg.). 2. Bd. Mexico Stadt: 1967

${ }^{4}$ Riese B. Aztekische Chrestomathie. Text LXII Xipe Totec, SAH01K18. 1996

${ }^{5}$ de Sahagún B. Primeros Memoriales (Farbfaksimile der Handschrift). Norman: University of Oklahoma Press, 1993

${ }^{6}$ Quilter J. Kunstschätze. In: Azteken-Ausstellungskatalog der Kunstund Ausstellungshalle der BRD. Bonn: 2003-2004

${ }^{7}$ Riese B. Aztekische Chrestomathie. Aztekische Religion und Kunst. 9. Stunde: Xipe. XIPEV. 1993

${ }^{8}$ Locke A. Gottheiten des Lebens. In: Azteken-Ausstellungskatalog der Kunst- und Ausstellungshalle der BRD. Bonn: 2003-2004

${ }^{9}$ Xipe Totec Encyclopaedia Britannica Delux Edition 2004, CD-ROM

${ }^{10}$ Moctezuma EM. Templo Mayor, der große Tempel der Azteken. In: Azteken-Ausstellungskatalog der Kunst- und Ausstellungshalle der BRD. Bonn: $2003-2004$

11 Libro de la Vida. Graz: Akad. Druck- und Verlagsanstalt, 1970

${ }^{12}$ Riese B. Aztekische Chrestomathie, Altmexikanische Heilkunde und Gesundheitspflege. Zeugnisse des 16. Jahrhunderts. A:|AZTGES 01|MMTEXT. 1996

Prof. Dr. Kurt Wegener

Pathologe

Wielandtstraße 14

69120 Heidelberg

E-mail: wegener@netclub.de 